\section{Obesity and Reproductive Health}

Philip Baker, Adam Balen, Lucilla Poston and

Naveed Sattar, eds.

ISBN: 978-1-9047523-9-4

London: RCOG Press, 2007

Obesity has reached epidemic proportions globally, with more than one billion adults classified as overweight, of whom at least 300 million are clinically obese. It is certainly a major contributor to the global burden of chronic disease and disability. Rates of obesity have risen nearly fourfold over the past quarter of a century and it is currently estimated that one in four adult women is obese.

In recent times, it has been recognized that the growing rates of obesity raise important public health issues relating to women and reproductive health. Specifically, there are a plethora of issues relating to the long-term metabolic sequelae for the women and their offspring, adverse pregnancy outcomes, infertility and rising rates of endometrial, ovarian and breast cancers.

This book, which is a product of the pioneering 53rd RCOG Study Group, is an excellent and comprehensive review into each of the areas relating to obesity and reproductive health. It collates all the evidence that is available to date and an expert in each field writes a critical appraisal of the area while emphasizing the urgent need for ongoing and future research on prevention and treatment. It stresses the need for the prevention of childhood obesity, which will exacerbate the epidemic in the future. There is also compelling data that a suboptimal maternal metabolic environment will have longterm implications for the offspring through the concept of 'fetal programming'. The final chapter in the book provides a summary of the consensus views, which arose from the Study Group - among which are clinically relevant treatment strategies in reducing the risk of pre-eclampsia, venous thromboembolic disease and congenital anomalies, all of which are more prevalent in obese pregnant women.

There is also an excellent chapter on 'Eating Disorders and Effects on Pregnancy', which summates all the available data in this field that has typically not been readily accessible to internists and obstetricians in the past. The chapters on 'Anti-obesity Surgery', 'Role of Drugs for Obesity' and 'Obesity and Anaesthesia' are very concise and provide an excellent reference for physicians and obstetricians who are not routinely exposed to these fields in their clinical practice.

In my opinion, this book is a must-have on the shelves of all training and practising obstetricians and physicians who have an active interest in the pre-conceptual counseling and medical care of pregnant women.

\section{Kenneth K Chen}

Consultant Endocrinologist, Fellow in Obstetric Medicine, Warren Alpert Medical School, Brown University, Providence, RI, USA

Email: kchen@mail.usyd.edu.au

DOI: 10.1258/om.2008.080055 